20

\title{
Экспериментально обучаемый метод фильтрации шумов рассеяния в сигналах оптической когерентной томографии*
}

\author{
(C) И.Н. Долганова ${ }^{1,2}$, Н.В. Черномырдинн ${ }^{2,3}$, П.В. Александрова ${ }^{2}$, И.В. Решетов ${ }^{4}$, В.Е. Карасик ${ }^{2}$, \\ К.И. Зайцев ${ }^{2,3}$, В.В. Тучин ${ }^{5}$ \\ ${ }^{1}$ Институт фоизики твердого тела РАН, \\ 142432 Черноголовка, Московская обл., Россия \\ ${ }^{2}$ Московский государственный технический университет им. Н.Э. Баумана, \\ 105005 Москва, Россия \\ ${ }^{3}$ Институт общей физики им. А.М. Прохорова Российской академии наук, \\ 119991 Москва, Россия \\ ${ }^{4}$ Первый Московский государственный медицинский университет им. И.М. Сеченова, \\ 119991 Москва, Россия \\ ${ }^{5}$ Саратовский национальный исследовательский государственный университет им. Н.Г. Чернышевского, \\ 410012 Саратов, Россия \\ e-mail: in.dolganova@gmail.com, e-mail: valeriykarasik@gmail.com
}

Поступила в редакцию 03.12.2018г.

В окончательной редакции 31.01.2019 г.

Принята к публикации 31.01.2019 г.

Описан метод обучения алгоритма вейвлетной фильтрации изображений оптической когерентной томографии (ОКТ) с помощью экспериментальных измерений тестовых объектов, изготовленных на основе водных растворов монодисперсных наночастиц и содержащих микроскопические включения. Выбор параметров тестовых объектов (концентрации раствора, размера наночастиц, формы, размера и взаимного положения включений) позволяет моделировать различные условия работы ОКТ-системы и устанавливать критерии оценки эффективности метода и параметров фильтрации ОКТ-изображений. В настоящей работе оптимальный фильтр для примера тестового объекта выбирался среди комбинаций различных базисных функций пяти семейств вейвлетов, „мягкого“ и „жесткого“ методов пороговой фильтрации, четырех уровней декомпозиции сигнала, а также значений порога фильтрации в диапазоне 0.05-3.05. Взаимное положение микровключений использовалось в качестве критерия оценки эффективности фильтрации. В результате показано, что установленный с помощью предложенного метода вейвлетный фильтр позволяет эффективно подавить шумы рассеяния ОКТ-изображений и сохранить информацию о структуре исследуемого объекта.

DOI: $10.21883 /$ OS.2019.05.47669.18-19

\section{Введение}

Оптическая когерентная томография является одним из перспективных методов визуализации внутренней структуры биологических тканей, который используется для методов „label-free“ диагностики в таких областях, как офтальмология, нейро- и сосудистая хирургия, онкология, дерматология и др. [1-12]. В ее основе лежит метод низкокогерентной интерферометрии [13-18], который позволяет получать изображения объектов (В-сканы, представляющие собой двумерное распределение интенсивности отраженного излучения от различных по глубине слоев объекта) с аксиальным разрешением порядка $5 \mu \mathrm{m}$, что значительно превосходит возможности визуализации внутренней структуры биотканей с помощью ультразвуковых приборов. Фор-

\footnotetext{
* The 22nd Annual Conference Saratov Fall Meeting 2018 (SFM'18): VI International Symposium, „Optics and Biophotonics“ and XXII International School for Junior Scientists and Students on Optics, Laser Physics \& Biophotonics, September 24-29, 2018, Saratov, Russia. https://www.sgu.ru/structure/fiz/saratov-fall-meeting/previousconferences/sara
}

мирование В-сканов производится чаще всего за счет сканирования в поперечном направлении, т. е. последовательной регистрации одномерных сигналов (А-сканов), за исключением так называемых „ОКТ полного поля““ Однако глубина зондирования ОКТ ограничена несколькими миллиметрами в зависимости от типа исследуемой ткани $[13,17,19]$. Причиной этому является сильное ослабление оптического излучения при распространении в биологической ткани, главным образом, за счет рассеяния на ее внутренних неоднородностях. Наличие шума рассеяния на ОКТ-изображениях препятствует более точному анализу структурных особенностей объектов, определению патологических состояний тканей и границ патологических включений $[20,21]$.

Существуют различные методы фильтрации шумов рассеяния в ОКТ-сигналах [22-31]. Среди них широкое распространение получили методы накопления сигналов, выделения полезной составляющей сигнала с помощью априорной оценки параметров и модели шума, цифровой фильтрации (медианной, винеровской, билатеральной и пороговой), а также вейвлетной фильтрации. Эффективность последней обусловлена тем, что 
базисные функции вейвлет-преобразования имеют схожие свойства с волновыми пакетами: нулевое среднее значение, ограниченность, а также локализация одновременно во временной и частотной областях. Применение вейвлетной фильтрации для ОКТ во многом объясняется возможностью эффективного подавления шума в изображениях, содержащих разномасштабные элементы, возможностью подавления спекл-шума и сглаживания изображения без существенного размытия деталей.

Построить вейвлетный фильтр можно с помощью различных базисных функций, а также параметров и методов фильтрации. Наличие большого числа примеров использования их разнообразных комбинаций не позволяет однозначно отдать предпочтение конкретному фильтру. Оценить эффективность различных фильтров на основе проведения измерений можно при условии, если точно известны параметры объекта, что в биомедицинских исследованиях зачастую возможно только при работе in vitro. Поэтому выбор параметров фильтра для конкретного объекта и ОКТ-системы представляет достаточно трудную задачу.

Одним из возможных путей ее решения является экспериментальная „настройка“ с использованием тестовых объектов параметров фильтрации: вейвлетного базиса, значения порога фильтрации, уровней декомпозиции сигнала. Учитывая широкий диапазон областей применения ОКТ, тестовый объект должен быть выбран в соответствии с условиями и особенностями исследования. Биологические ткани представляют собой сильно рассеивающие среды, поэтому удобно в качестве тестовой среды использовать монодисперсные коллоидные суспензии наночастиц, рассеивающие параметры которых можно изменять с помощью выбора диаметра частиц и их концентрации, что предоставляет определенную гибкость в выборе модельных сред. В зависимости от задачи исследований можно выбрать структуру тестового объекта. Например, при необходимости дифференциации типов тканей по коэффициенту рассеяния, либо для восстановления границы раздела (как в случае определения областей злокачественного поражения) тестовый объект может иметь слоистую структуру, а для исследования наличия микровключений и особенностей структуры ткани (как в случае определения положения внутридермальных невусов или наличия участков некроза тканей) объект может иметь дополнительные элементы известной формы и положения. На основе имеющейся априорной информации об объекте и его структуре можно сформировать критерий оценки эффективности вейвлетных фильтров, с помощью которого может быть выбран оптимальный фильтр, учитывающий как параметры ОКТ-системы, так и особенности исследуемого объекта.

В настоящей работе продемонстрирован пример использования такого подхода для анализа полубесконечной рассеивающей среды с микровключениями, приведено описание тестового объекта и алгоритма вы- бора параметров вейвлетной фильтрации. Рассматривались фильтры, образованные 52 материнскими функциями, „жестким“ и „мягким“ методом пороговой фильтрации, четырьмя уровнями декомпозиции сигнала и 61 значением порога фильтрации, в качестве критерия оценки эффективности фильтра использовалось соответствие информации о взаимном положении структурных неоднородностей объекта до и после фильтрации. Также проиллюстрирована работа выбранного фильтра в условиях анализа структуры реального биологического объекта. Выбор среди различных значений порога фильтрации позволил уточнить параметры фильтра по сравнению с ранее полученными результатами для стандартных значений порога [32], что демонстрируют полученные изображения структуры биологической среды.

\section{Тестовый объект}

В соответствии с предложенным критерием был изготовлен тестовый объект, показанный на рис. 1 и состоящий из трех медных проволок диаметром $80 \mu \mathrm{m}$, расположенных параллельно друг другу в стеклянной кювете, заполненной водным раствором монодисперсных наночастиц с концентрацией $0.25 \%$, в качестве которых были использованы частицы аморфного кремния диаметром $400 \mathrm{~nm}$, изготовленные методом Штобера [33,34]. Концентрация раствора была выбрана произвольно для демонстрации предлагаемого подхода. Проволоки жестко фиксировались в кювете, расстояния между ними были измерены с помощью микроскопа и равнялись $r_{1}=0.5 \mathrm{~mm}$ и $r_{2}=0.4 \mathrm{~mm}$. Априорная информация о тестовом объекте, а именно отношение расстояний между проволоками $r_{1} / r_{2}$, использовалась для анализа эффективности фильтрации его ОКТ-изображений. Следует отметить, что возможность изменения концентрации раствора позволяет предварительно выбирать вейвлетный фильтр для исследования биологического объекта, точные данные о параметрах которого отсутствуют.

Были получены несколько ОКТ-изображений описанного выше тестового объекта с помощью коммерческой системы ОКТ 1300У. Данная система работает в ближнем ИК диапазоне, центральная длина волны источника системы составляет $1300 \mathrm{~nm}$. С ее помощью регистрируются двумерные изображения (В-сканы) размером $256 \times 400$ пикселей с заявленным разрешением $50 \mu \mathrm{m}$ в латеральном и $30 \mu \mathrm{m}$ в аксиальном направлениях в воздухе.

Из рис. 1, $c$ видно, что наличие рассеивающей среды - раствора наночастиц - приводит к существенным искажениям изображения и появлению шума, из-за чего становится сложнее определить положения центров включений. Смещения по вертикали положений частиц обусловлены неточным позиционированием ОКТ-зонда по отношению к объекту, а именно его наклоном. 


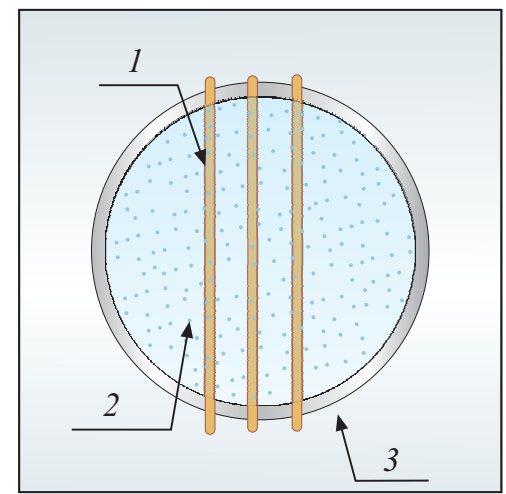

$a$
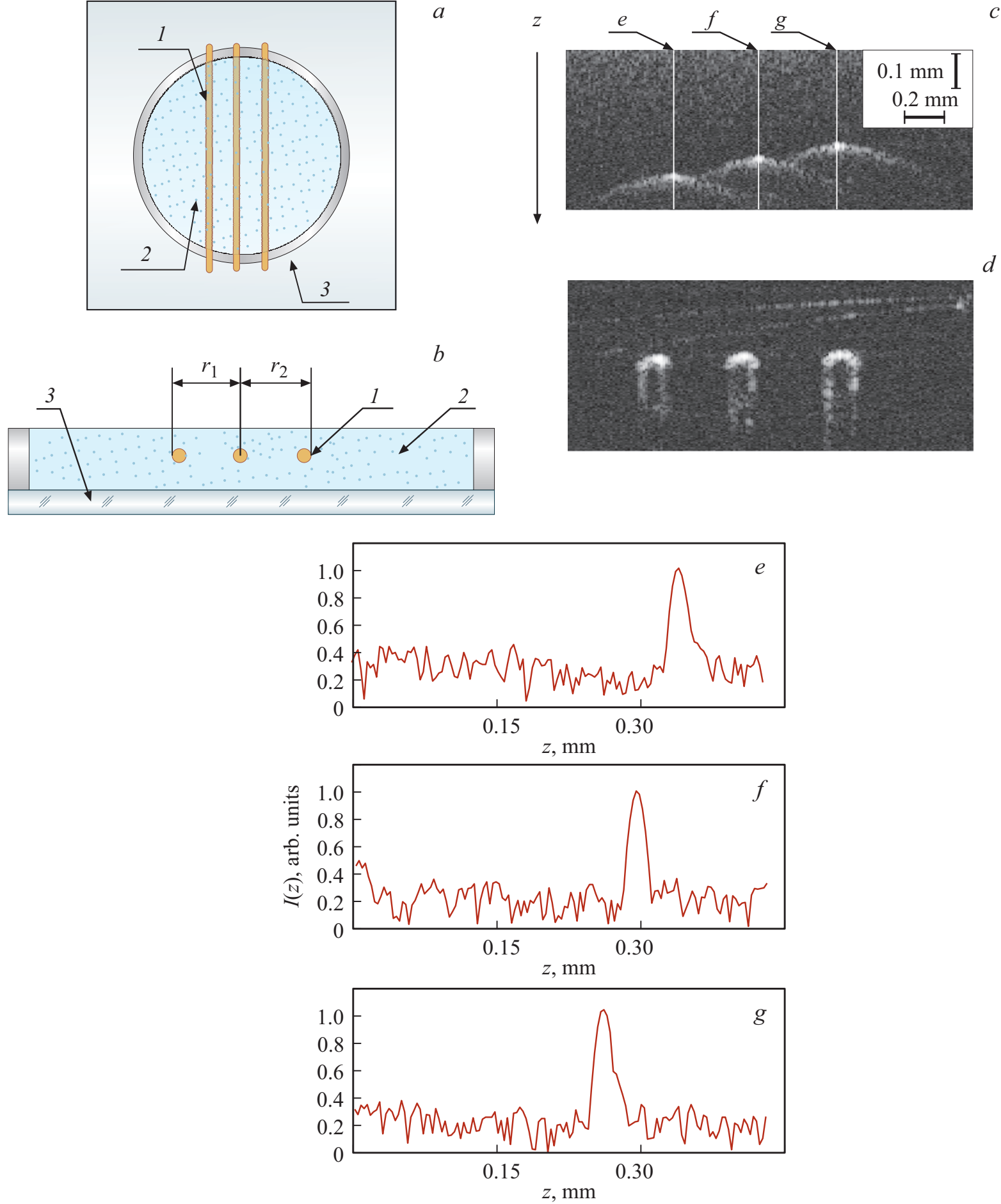

Рис. 1. Схема тестового объекта и регистрируемые сигналы ОКТ: $a$ - схема тестового объекта (вид сверху), где 1 микровключения (проволоки), 2 - рассеивающие частицы водного раствора, 3 - покровное стекло; $b-$ боковое сечение схемы тестового объекта, $r_{1}$ и $r_{2}-$ расстояния между центрами микровключений; $c-$ пример исходного ОКТ-изображения объекта $(\mathrm{B}-с к а н) ; d-$ ОКТ-изображение исходного объекта в отсутствие рассеивающей среды (в воздухе); $e, f, g$ - вертикальные сечения ОКТ сигнала (А-сканы), соответствующие положениям центров микровключений.

В биологических средах наряду с рассеянием на структуре ткани поглощение и рассеяние на поверхности объекта также могут вносить определенные искажения в формируемый ОКТ-сигнал. Процедура вейвлетной филь- трации при условии выбора оптимальных параметров способствует подавлению шумов ОКТ-сигнала (В-скана), обусловленных указанными факторами, при этом может обеспечить сохранение информации об объекте 
исследования, а именно о его внутренней структуре и распределении коэффициента рассеяния [32].

\section{Алгоритм выбора параметров фильтра}

Выбор оптимальных параметров вейвлетного фильтра осуществляется на основе полученных ОКТизображений тестового объекта, априорных данных и алгоритма, описанного ниже.

Для отдельных А-сканов (интенсивность отраженного излучения в фиксированной точке объекта $x^{\prime}$ ) $I_{X}(z)=I\left(x=x^{\prime}, z\right)$, где $z$ - глубина объекта, $x$ - латеральная (поперечная) координата, процедуру вейвлетной фильтрации можно осуществить с помощью прямого и обратного вейвлет-преобразования, а также выбора порогового значения $T$.

Прямое вейвлет-преобразование описывается соотношением

$$
C(a, b)=W[I(z)]=\int_{-\infty}^{\infty} I_{X}(z) \psi(a, b, z) d z,
$$

где $\psi(z)$ - материнский вейвлет, а $\psi(a, b, z)-$ ядро вейвлет-декомпозиции с параметрами масштаба и смещения $a$ и $b$ соответственно:

$$
\psi(a, b, z)=|a|^{-1 / 2} \psi\left(\frac{z-b}{a}\right) .
$$

Обратное вейвлет-преобразование с учетом порога:

$$
\begin{gathered}
I^{\prime}(z)=W^{-1}\left[C_{T}(a, b)\right]=C_{\psi} \iint_{-\infty}^{\infty} \frac{C_{T}(a, b)}{a^{2}} \tilde{\psi}(a, b, z) d a d b, \\
C_{T}(a, b)= \begin{cases}C(a, b), & \text { если } C(a, b) \geq T, \\
0, & \text { если } C(a, b)<T,\end{cases} \\
C_{\psi}=\int_{-\infty}^{\infty} \frac{\Psi(\omega) \tilde{\Psi}(\omega)}{|\omega|} d \omega<\infty,
\end{gathered}
$$

где $\Psi(\omega)$ и $\tilde{\Psi}(\omega)$ - фурье-образы $\psi(z)$ и $\tilde{\psi}(z)$ соответственно, $\tilde{\psi}(z)$ - функция, сопряженная с $\psi(z)$.

Выражения (1)-(5) следует применить для каждого полученного ОКТ-изображения тестового объекта, варьируя при этом параметры фильтров. В настоящей работе использовались различные вейвлетные базисные функции, „мягкая““ $\left(, s^{\text {“ }}\right)$ и „жесткая“ $\left(, h^{\text {“ }}\right)$ пороговая фильтрация (4) [35], а также значения порога фильтрации в диапазоне 0.05-3.05 с шагом 0.05. Для данного диапазона характерны вариации ошибки результатов фильтрации более $0.01 \%$. Для ускорения вычислений были использованы алгоритмы быстрого прямого и обратного вейвлет-преобразований [36] и соответственно различные уровни декомпозиции сигнала.
На полученных отфильтрованных изображениях необходимо выделить с помощью, например, пороговой фильтрации по значению $I^{\prime}(x, z)$ сегменты с максимальным значением интенсивности, которые соответствуют изображениям микровключений, а также найти их центры масс по интенсивности [37]. Их относительное положение $\left(r_{1} / r_{2}\right)_{F}$ в сравнении с априорным значением $\left(r_{1} / r_{2}\right)_{0}$ составляет ошибку фильтрации каждого $i$-го ОКТ-изображения тестового объекта:

$$
d_{i}=\left|\left(r_{1} / r_{2}\right)_{F}-\left(r_{1} / r_{2}\right)_{0}\right|
$$

Среднеквадратичное отклонение (СКО) ошибки для серии из $N$ изображений тестового объекта определяет эффективность использованного фильтра:

$$
\sigma_{\mathrm{STD}}=\left(\frac{1}{N} \sum_{i=1}^{N} d_{i}^{2}\right)^{1 / 2} .
$$

В настоящей работе были получены $N=5$ ОКТизображений. Минимальное значение $\sigma_{\mathrm{STD}}$ позволяет определить подходящий вейвлетный фильтр для данных конкретных условий: параметров ОКТ-системы и выбранных рассеивающих и геометрических параметров объекта.

\section{Результаты}

Описанный алгоритм был использован для анализа 25376 вейвлетных фильтров, образованных 52 материнскими функциями, принадлежащих пяти семействам (daubechies („db“), coiflets („,coif“), symlets (,,sym“"), biorthogonal („,bior ${ }^{\text {“6 }}$ ), reverse biortogonal $($,rbio“) ), двумя методами пороговой фильтрации (,мягкой“6 и „жесткой“6), четырьмя уровнями декомпозиции сигнала $(L=2,4,6,8)$ и 61 значением порога фильтрации. Вычисления были выполнены с помощью пакета MatLab с использованием встроенных вейвлетных функций.

На рис. 2 показано распределение значений ошибки $(7)$ в логарифмической шкале $\log \left(1+\sigma_{\mathrm{STD}}\right)$, полученное для тестового объекта. Видно, что чем больше уровень декомпозиции сигнала, тем большая наблюдается зависимость от выбора значения порога фильтрации $T$. В свою очередь, зависимость от изменения порога носит более плавный характер для „мягкого“ метода. Среди всех рассмотренных комбинаций для используемого тестового объекта минимальным значением ошибки $\log \left(1+\sigma_{\mathrm{STD}}\right)=0.005$ обладает фильтр „,bior3.5“ $(L=4$, $h, T=0.65)$, отмеченный на рис. 2 стрелкой.

Следует отметить, что при использовании стандартного метода определения порога фильтрации $T=(2 \ln (n))^{1 / 2}\langle C\rangle / 0.6745$, где $n$ - число элементов изображения, $\langle C\rangle$ - среднее значение коэффициентов разложения, ранее было показано, что фильтр „bior3.5“ $(L=4, h, T=0.306)$ также позволяет получить наименьшую ошибку определения параметров структуры 

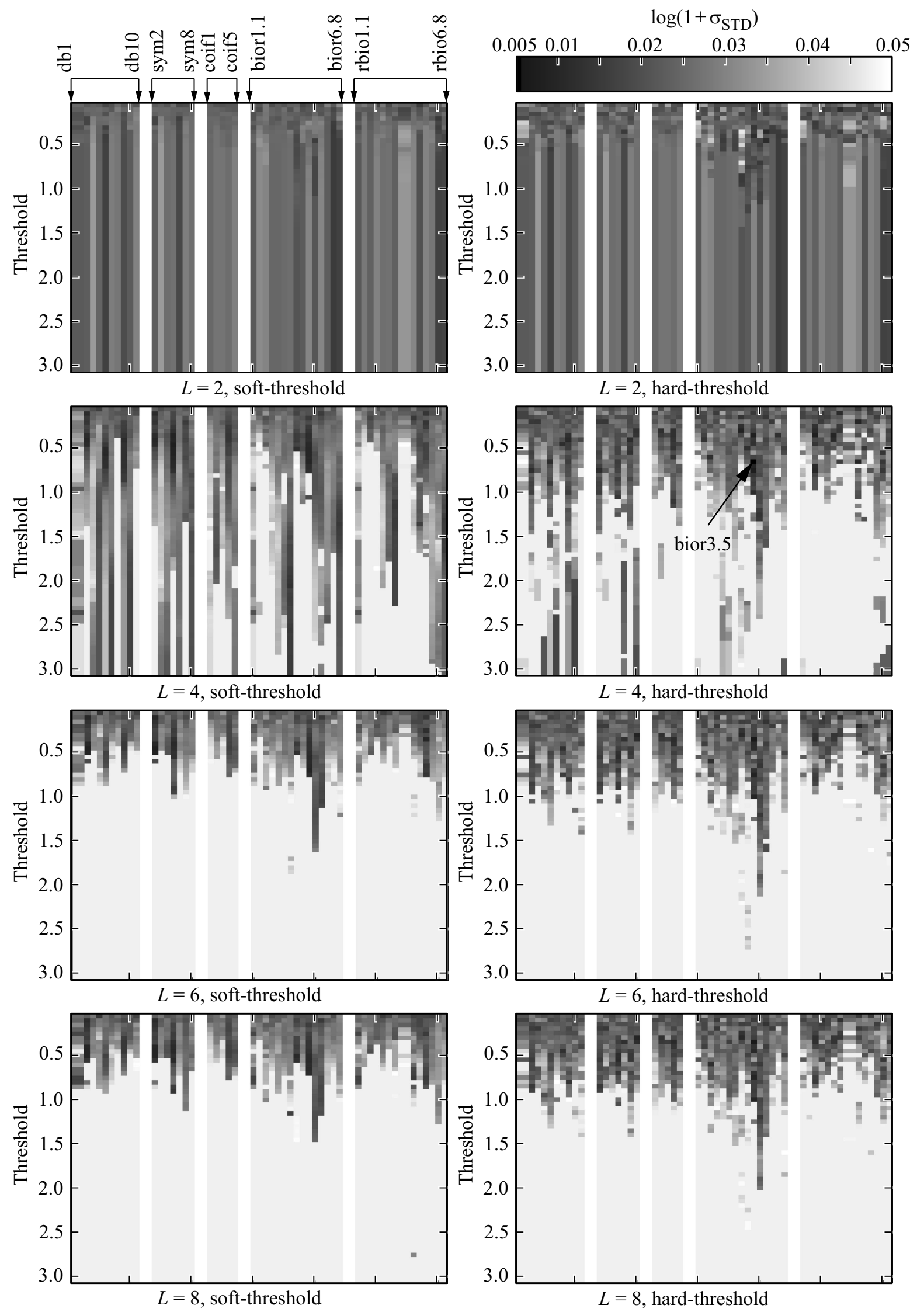

Рис. 2. Распределение значений ошибки использования различных вейвлетных фильтров: слева — „мягкая“ пороговая фильтрация, справа - „жесткая“; $L$ - уровень декомпозиции. 
$a$

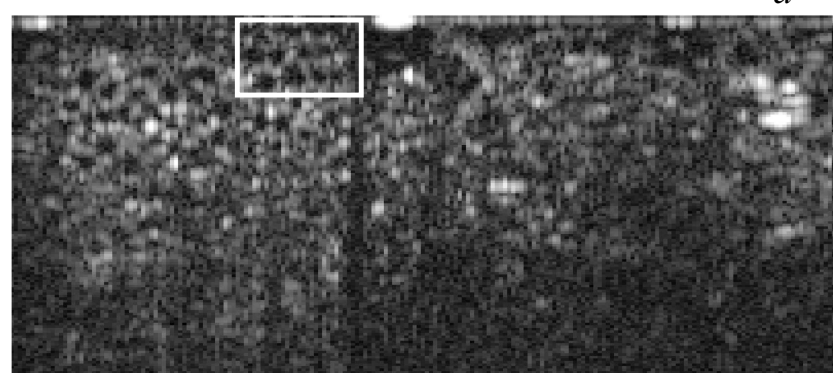

$c$

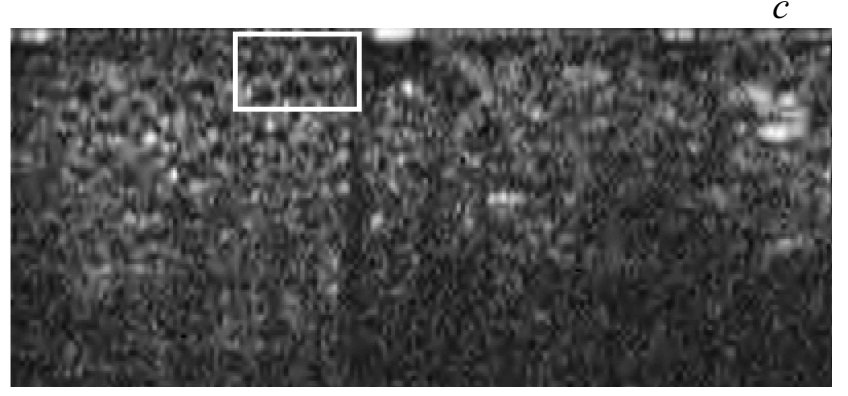

$e$

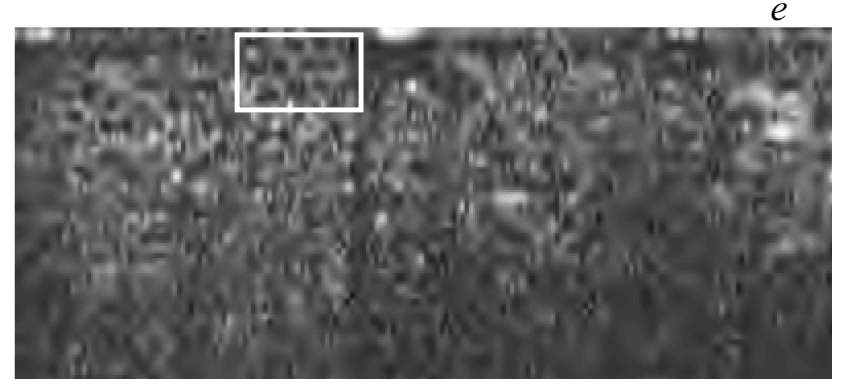

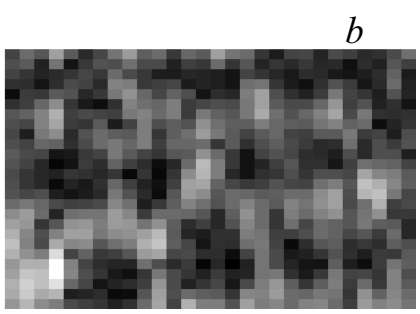
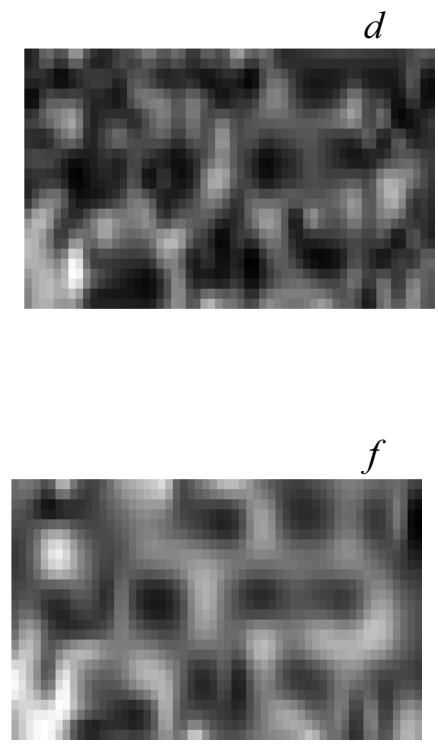

Рис. 3. Результат использования вейвлетных фильтров: $a-$ исходное изображение объекта; $c-$ результат использования фильтра „bior3.5“ $(L=4, h, T=0.306) ; e-$ результат использования фильтра „bior $3.5^{\text {“ }}(L=4, h, T=0.65) ; b, d, f-$ увеличенные изображения отдельных участков на $a, c, e$ соответственно.

объекта [32]. При этом $\log \left(1+\sigma_{\mathrm{STD}}\right)=0.01$, таким образом, с помощью подбора значения порога фильтрации вручную удается снизить значение ошибки в два раза.

Чтобы оценить эффективность выбранных параметров вейвлетной фильтрации, была проанализирована работа фильтра для изображения объекта - мякоти банана, имеющего мелкую структуру, состоящую из клеток размерами порядка $50 \mu \mathrm{m}$ (рис. 3). На рис. 3, $a$ показано исходное ОКТ-изображение данного объекта, где видно, что он содержит множество мелких включений. При этом большие трудности вызывает определение как формы, так и границ этих включений из-за сильной зашумленности изображения. На рис. $3, c$ показан результат использования фильтра „bior3.5“ $(L=4, h, T=0.306)$ без выбора порога, а на рис. 3,e - результат использования выбранного в данной работе фильтра „bior $3.5^{\prime}$ $(L=4, h, T=0.65)$ с уточненным значением порога. На увеличенных участках изображений заметно, что подавление шума более эффективно выполнено для выбранного фильтра с сохранением информации о положении и форме включений, о чем свидетельствует восстановление границ включений, имеющих разрывы на исходном изображении.

Следует отметить, что в зависимости от точности имеющихся начальных данных об объекте исследования диапазон значений параметров тестового объекта может различаться (размеры и форма частиц, концентрация и размеры рассеивателей). Это будет влиять на количество обращений к описанному алгоритму. В частности, как было показано ранее [32], можно использовать несколько концентраций коллоидного раствора наночастиц для определения эффективного фильтра в широком диапазоне параметров рассеяния. А при известных исходных данных об исследуемом объекте может потребоваться лишь одна конфигурация тестового объекта. При этом увеличение диапазона значений параметров приводит к нахождению более универсального фильтра для ОКТвизуализации.

Для выбора оптимальных параметров вейвлетной фильтрации требуется анализ большого числа их комбинаций, что ведет к использованию значительных затрат расчетного времени. В частности, при получении 
описанных в данной работе результатов с помощью стандартного персонального компьютера время выбора оптимального фильтра составило около $1 \mathrm{~h}$. При оптимизации алгоритма расчета с использованием параллельных методов вычислений можно сократить это значение, но тем не менее проведение подобной процедуры, например, во время интраоперационной диагностики, будет существенно влиять на общее время диагностики. Предложенный метод настройки параметров вейвлетного фильтра может быть использован заранее, что приведет к подготовке одного или нескольких фильтров для дальнейшего применения и не потребует существенного дополнительного времени для анализа ОКТ-сигналов в ходе исследования.

Предлагаемый в настоящей работе вейвлетный фильтр и критерий выбора параметров фильтрации может быть использован для анализа структуры объекта с мелкими включениями. Однако важно заметить, что при необходимости исследования прочих объектов, имеющих другую структуру, критерий оценки эффективности вейвлетного фильтра может быть изменен так же, как и тестовый объект.

\section{Заключение}

В настоящей работе продемонстрирован алгоритм выбора оптимального вейвлетного фильтра для ОКТвизуализации различных объектов, основанный на экспериментальном исследовании тестового объекта. В качестве тестового объекта был предложен водный раствор наночастиц, содержащий микровключения - металлические проволоки. Априорные данные об объекте (о его рассеивающих и геометрических параметрах) использовались для построения критерия оценки вейвлетных фильтров. Выбор объекта и критерия зависят от поставленной задачи и могут изменяться. Оценка эффективности использования большого количества вейвлетных фильтров позволяет подстроить параметры фильтра под данные конкретные условия, либо под диапазон условий. Описанный подход может использоваться для большого спектра приложений ОКТ-визуализации, в частности, для задач биомедицины, когда априорные данные об объекте исследования отсутствуют.

\section{Финансирование работы}

Работа выполнена при финансовой поддержке РНФ в рамках научного проекта № 14-19-01083.

\section{Список литературы}

[1] Huang D., Swanson E.A., Lin C.P., Schuman J.S., Stinson W.G., Chang W., Hee M.R., Flotte T., Gregory K., Puliafito C.A. // Science. 1991. V. 254. N 5035. P. $1178-$ 1181 doi 10.1126/science.1957169
[2] Puliafito C.A., Hee M.R., Lin C.P., Reichel E., Schuman J.S., Duker J.S., Izatt J.A., Swanson E.A., Fujimoto J.G. // Ophthalmology. 1995. V. 102. N 2. P. 217-229. doi 10.1016/S0161-6420(95)31032-9

[3] Fujimoto J.G. // Nature Biotechnology. 2003. V. 21. N 11. P. $1361-1367$. doi $10.1038 /$ nbt 892

[4] Aum J., Kim J., Jeong J. // Appl. Opt. 2015. V. 54. P. D43D50. doi 10.1364/AO.54.000D43

[5] Lichtenegger A., Harper D.J., Augustin M., Eugui P., Muck M., Gesperger J., Hitzenberger C.K., Woehrer A., Baumann B. // Biomed. Opt. Expr. 2016. V. 8. N 9. P. $4007-$ 4025. doi. 10.1364/BOE.8.004007

[6] Baran U., Wang R.K. // Neurophotonics. 2016. V. 3. N 1. P. 010902 doi 10.1117/1.NPh.3.1.010902

[7] Yashin K.S., Kiseleva E.B., Gubarkova E.V., Matveev L.A., Karabut M.M., Elagin V.V., Sirotkina M.A., Medyanik I.A., Kravets L.Ya., Gladkova N.D. // Proc. SPIE. 2017. V. 10050. P. 10050Z. doi 10.1117/12.2252286

[8] Yabushita H., Bouma B.E., Houser S.L., Aretz H.T., Jang I.-K., Schlendorf K.H., Kauffman C.R., Shishkov M., Kang D.-H., Halpern E.F., Tearney G.J. // Circulation. 2002. V. 106. N 13. P. 1640-1645. doi 10.1161/01.CIR.0000029927.92825.F6

[9] Winetraub Y., SoRelle E.D., Liba O., de la Zerda A. // Appl. Phys. Lett. 2016. V. 108. N 2. P. 023702. doi 10.1063/1.4939547

[10] Welzel J. // Skin Research Technology. 2001. V. 7. N 1. P. 1-9. doi 10.1034/j.1600-0846.2001.007001001.x

[11] Zarnescu L., Leung M.C., Abeyta M., Sudkamp H.M., Baer T.M., Behr B., Ellerbee A.K. // J. Biomedical Optics. 2015. V. 20. N 9. P. 096004. doi 10.1117/1.JBO.20.9.096004

[12] Kut C., Chaichana K.L., Xi J., Raza S.M., Ye X., McVeigh E.R., Rodriguez F.J., Quiñones-Hinojosa A., Li X. // Science Translation Medicine. 2015. V. 7. N 292. P. 292ra100. doi 10.1126/scitranslmed.3010611

[13] Tuchin V.V. Handbook of Coherent-Domain Optical Methods. N.Y.: Springer, 2013.

[14] Wojtkowski M. // Appl. Opt. 2010. V. 49. N 16. P. D30-D61. doi 10.1364/AO.49.000D30

[15] Tearney G.J., Brezinski M.E., Bouma B.E., Boppart S.A., Pitris C., Southern J.F., Fujimoto J.G. // Science. 1997. V. 276. N 5321. P. 2037-2039. doi 10.1126/science.276.5321.2037

[16] Drexler W., Morgner U., Kärtner F.X., Pitris C., Boppart S.A., Li X.D., Ippen E.P., Fujimoto J.G. // Opt. Lett. 1999. V. 24. N 17. P. 1221-1223. doi 10.1364/OL.24.001221

[17] Tuchin V.V. Tissue Optics: Light Scattering Methods and Instruments for Medical Diagnostics. Bellingham, Washington: SPIE Press, 2015.

[18] Вишняков Г.Н., Левин Г.Г., Минаев В.Л., Ермаков М.М. // Опт. и спектр. 2018. Т. 125. № 6. С. 864. doi 10.21883/OS.2018.12.46952.155-18

[19] Dolganova I.N., Neganova A.S., Kudrin K.G., Zaytsev K.I., Reshetov I.V. // J. Phys. Conf. Ser. 2016. V. 673. P. 012014. doi 10.1088/1742-6596/673/1/012014

[20] Schmitt J.M., Xiang S.H., Yung K.M. // J. Biomed. Opt. 1999. V. 4. N 1. P. 95-105. doi 10.1117/1.429925

[21] Almasian M., van Leeuwen T.G., Faber D.J. // Sci. Rep. 2017. V. 7. P. 14873. doi 10.1038/s41598-017-14115-3

[22] Baghaie A., D’Souza R.M., Yu Z. // Optik. 2016. V. 127. N 15. P. 5783-5791. doi 10.1016/j.ijleo.2016.03.078

[23] Steiner P., Kowal J.H., Považay B., Meier C., Sznitman R. // Appl. Optics. 2015. V. 54. P. 36503. doi 10.1364/AO.54.003650 
[24] Kim K.-S., Park H.-J., Kang H.S. // Opt. Engin. 2015. V. 54. N 11. P. 113110. doi 10.1117/1.OE.54.11.113110

[25] Adler D.C., Ko T.H., Fujimoto J.G. // Opt. Lett. 2004. V. 29. P. 2878. doi 10.1364/OL.29.002878

[26] Buranachai C., Thavarungkul P., Kanatharana P., Meglinski I.V. // Laser Phys. Lett. 2009. V. 6. N 12. P. 892-895. doi 10.1002/lapl.200910089

[27] Mayer M.A., Borsdorf A., Wagner M., Hornegger J., Mardin C.Y., Tornow R.P. // Biomedical Optics Express. 2012. V. 3. P. 572. doi 10.1364/BOE.3.000572

[28] Pavlov A.N., Nazimov A.I., Pavlova O.N., Lychagov V.V., Tuchin V.V., Bibikova O.A., Sindeev S.S., SemyachkinaGlushkovskaya O.V. // J. Innovative Optical Health Sciences. 2014. V. 7. N 1. doi 10.1142/S1793545813500557

[29] Dolganova I.N., Aleksandrova P.V., Beshplav S.-I.T., Chernomyrdin N.V., Dubyanskaya E.N., Goryaynov S.A., Kurlov V.N., Reshetov I.V., Potapov A.A., Tuchin V.V., Zaytsev K.I. // Proc. SPIE. 2018. V. 10717. P. 107171X. doi 10.1117/12.2314727

[30] Zaytsev K.I., Kudrin K.G., Chernomyrdin N.V., Khorokhorov A.M., Prytov A.B., Dolganova I.N., Perchik A.V., Reshetov I.V., Yurchenko S.O. // J. Phys. Conf. Ser. 2015. V. 584. P. 012013.

[31] Chernomyrdin N.V., Zaytsev K.I., Gavdush A.A., Fokina I.N., Karasik V.E., Reshetov I.V., Kudrin K.G., Nosov P.A., Yurchenko S.O. // Proc. SPIE. 2014. V. 9216. P. 921611. doi $10.1117 / 12.2061276$

[32] Dolganova I.N., Chernomyrdin N.V., Aleksandrova P.V., Beshplav S.-I.T., Potapov A.A., Reshetov I.V., Kurlov V.N., Tuchin V.V., Zaytsev K.I. // J. Biomed. Opt. 2018. V. 23. N 9. P. 091406. doi 10.1117/1.JBO.23.9.091406

[33] Stober W., Fink A., Bohn E. // J. Coll. Interf. Sci. 1968. V. 26. N 1. P. 62-69. doi 10.1016/0021-9797(68)90272-5

[55] Zhokhov A.A., Masalov V.M., Sukhinina N.S., Matveev D.V., Dolganov P.V., Dolganov V.K. P. 208-212. doi 10.1016/00219797(68)90272-5

[34] Zhokhov A.A., Masalov V.M., Sukhinina N.S., Matveev D.V., Dolganov P.V., Dolganov V.K., Emelchenko G.A. // Opt. Mater. 2015. V. 49. P. 208-212. doi 10.1016/j.optmat.2015.09.019

[36] Mallat S.G. // IEEE Trans. Pattern Analysis and Machine Intell. 1989. V. 11. N 7. P. 674-693. doi 10.1109/34.192463

[37] Gonzalez R.C., Woods R.E. Digital Image Processing. New Jersey: Prentice-Hall, 2006. 